\title{
Correlational Evidence for the Role of Spatial Perspective-Taking Ability in the Mental Rotation of Human-Like Objects
}

\author{
Hiroyuki Muto (1)
}

Kokoro Research Center, Kyoto University, Japan

\begin{abstract}
People can mentally rotate objects that resemble human bodies more efficiently than nonsense objects in the same/different judgment task. Previous studies proposed that this human-body advantage in mental rotation is mediated by one's projections of body axes onto a human-like object, implying that human-like objects elicit a strategy shift, from an object-based to an egocentric mental rotation. To test this idea, we investigated whether mental rotation performance involving a human-like object had a stronger association with spatial perspective-taking, which entails egocentric mental rotation, than a nonsense object. In the present study, female participants completed a chronometric mental rotation task with nonsense and human-like objects. Their spatial perspective-taking ability was then assessed using the Road Map Test and the Spatial Orientation Test. Mental rotation response times (RTs) were shorter for human-like than for nonsense objects, replicating previous research. More importantly, spatial perspective-taking had a stronger negative correlation with RTs for human-like than for nonsense objects. These findings suggest that human-like stimuli in the same/different mental rotation task induce a strategy shift toward efficient egocentric mental rotation.
\end{abstract}

Keywords: mental rotation, human-body analogy, embodiment, spatial perspective-taking, spatial transformation, spatial cognition

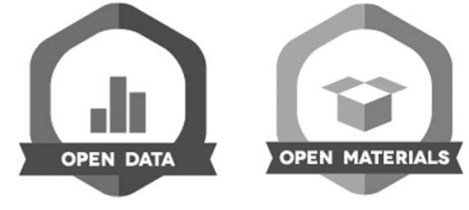

\section{Human-Body Advantage in Mental Rotation}

Object-based mental rotation refers to the ability to create a mental image of an object and then mentally rotate it. In a classic experiment, Shepard and Metzler (1971) presented participants with a pair of three-dimensional objects, consisting of cubes, and required them to judge whether the two objects were the same or mirror images of each other regardless of the objects' orientations. The typical results showed a linear increase in mean response time (RT) with the angular disparity between the two objects, suggesting that people can mentally rotate objects at a constant rate as they physically rotate real objects (for a recent review, see Searle \& Hamm, 2017).

People can perform a same/different mental rotation task more efficiently when the stimuli are human bodies
(Amorim et al., 2006; Jansen et al., 2012; Voyer \& Jansen, 2016) or cube objects with human-like features (e.g., head, face) that are analogous to human bodies (Amorim et al., 2006; Krüger et al., 2014; Makinae et al., 2015; Makinae \& Kasai, 2017; Muto et al., 2020; Muto \& Nagai, 2020; Sayeki, 1981; Voyer \& Jansen, 2016) compared with nonsense cube objects. Amorim et al. (2006) proposed two underlying processes in the human-body advantage in mental rotation, called "spatial embodiment" and "motoric embodiment." The spatial embodiment describes the process of projecting one's body axes onto human bodies or human-like objects with intrinsic axes (e.g., the top-bottom axis can be defined by the head position), while the motoric embodiment describes the process of the mental emulation of the posture of stimuli.

Empirical support for spatial and motoric embodiment remains limited. Accordingly, mental rotation performance did not improve when the stimuli were impossible postures (Amorim et al., 2006; Krüger et al., 2014; Sayeki, 1981) or desk lamps, which are familiar but not related to human bodies (Amorim et al., 2006). In addition, Jansen et al. (2012) reported that compared with non-athletes, male soccer players showed shorter overall 
RTs (but not mental rotation speeds) only for human bodies, which is at least consistent with a motoric embodiment. However, contrary to the motoric embodiment (and consistent with the spatial embodiment), Muto and Nagai (2020) demonstrated that snake-like objects, which had intrinsic axes but were difficult to imitate, increased mental rotation speeds by the same rate as the human-body advantage. Given the limited research and mixed results, more empirical investigations are needed to determine the mechanisms underlying the humanbody advantage. In particular, the present study focused on the spatial embodiment.

\section{Spatial Embodiment and Egocentric Mental Rotation}

Spatial embodiment raises the possibility that participants not only execute object-based mental rotation but also mentally transform their perspectives to align their imagined orientation with the stimulus orientation in a same/different judgment task with human-like stimuli, which was claimed by some researchers (Amorim et al., 2006; Jansen et al., 2012; Voyer \& Jansen, 2016). Such a perspective transformation has been considered as the mental rotation of the self, or egocentric mental rotation, which has a well-established distinction from object-based mental rotation (e.g., Habacha et al., 2018; Hegarty \& Waller, 2004; Huttenlocher \& Presson, 1973; Kessler \& Thomson, 2010; Kozhevnikov \& Hegarty, 2001; Muto et al., 2019, 2018; Wraga et al., 2000; Zacks et al., 2000; Zacks \& Michelon, 2005; Zacks \& Tversky, 2005). In most situations, compared with object-based mental rotation, egocentric mental rotation is more efficient and shows shallower RT slopes as a function of angular disparity (e.g., Habacha et al., 2018; Huttenlocher \& Presson, 1973; Voyer et al., 2017; Wraga et al., 2000; Zacks et al., 2000; Zacks \& Michelon, 2005; Zacks \& Tversky, 2005). Hence, one may partially attribute the human-body advantage to a strategy shift from the objectbased mental rotation to the more efficient egocentric mental rotation. Nonetheless, to the best of our knowledge, no studies have directly investigated the role of egocentric mental rotation in a same/different judgment task involving human-like stimuli.

\section{The Present Study}

The present study first investigated whether the egocentric mental rotation is adopted for the same/different comparison of human-like objects. Although egocentric mental rotation was shown to be adopted for left/right judgments of a rotated human body (e.g., Habacha et al., 2018; Zacks et al., 2000; Zacks \& Michelon, 2005; Zacks \& Tversky, 2005), it is controversial whether the adoption of egocentric mental rotation is determined by stimulus type (i.e., human-likeness) or task type (i.e., left/right identification; e.g., Voyer et al., 2017). In other words, it is still unclear whether the egocentric mental rotation is elicited for a same/different judgment of human-like objects. Thus, we examined whether the human-body advantage can be explained by egocentric mental rotation efficiency using an individual-differences approach as a first step.

Spatial perspective-taking, which refers to one's ability to understand an object's direction from a perspective other than their own, is known to entail egocentric mental rotation (e.g., Hegarty \& Waller, 2004; Kessler \& Thomson, 2010; Kozhevnikov \& Hegarty, 2001; Muto et al., 2018, 2019). Consistent with this, Zacks et al. (2000) showed that performance on left/right judgments of human bodies (requiring egocentric mental rotation) was more strongly associated with the spatial perspective-taking ability, assessed by psychometric tests, than object-based mental rotation. Hence, we hypothesized that spatial perspective-taking ability has a stronger association with performance on the same/ different judgment of human-like objects as opposed to nonsense ones.

To minimize the stimulus difference between the control and human-body conditions, the present study used cube objects as stimuli to which a human-like or nonsense pattern was added to appear or not appear as whole human bodies, respectively, following some previous studies (Makinae et al., 2015; Makinae \& Kasai, 2017; Muto \& Nagai, 2020; Sayeki, 1981), rather than using real human bodies (Amorim et al., 2006; Jansen et al., 2012; Voyer \& Jansen, 2016). In addition, we recruited only female participants given the previous findings of sex differences observed in both object-based and egocentric mental rotation (e.g., Makinae et al., 2015; Makinae \& Kasai, 2017; Tarampi et al., 2016; Voyer et al., 1995; Voyer et al., 2017; Voyer \& Jansen, 2016) and larger human-body advantage among females than males in chronometric mental rotation experiments with cube stimuli (Makinae \& Kasai, 2017; Makinae et al., 2015; but also see Voyer \& Jansen, 2016, who reported the opposite result in an experiment using real human bodies). To assess spatial perspectivetaking ability, we used the Road Map Test (Money et al., 1965, modified by Zacks et al., 2000) and the Spatial Orientation Test (Kozhevnikov \& Hegarty, 2001, modified by Hegarty \& Waller, 2004), which are commonly used as psychometric measures (e.g., Tarampi et al., 2016; Zacks et al., 2000). The main goal of the present study was to test whether human-like stimuli induce a strategy shift toward efficient egocentric mental rotation via spatial 
embodiment. If this hypothesis is true, spatial perspectivetaking ability should have a stronger negative correlation with mean RT for the same/different judgment of humanlike than nonsense objects.

\section{Methods}

\section{Participants}

Since the effect size was unknown, we patterned our sample size to that of Zacks et al. (2000), whose measures were similar to those of the present study and where data from 48 participants were analyzed. We planned to collect data from 50 women enrolled in an introductory psychology course (as part of a requirement). However, two did not participate in the experiment, one completed only the mental rotation task, and four had near-chance performance in at least one condition of the mental rotation task. Thus, only data from 43 women (mean age $=19.1$ years, ranging from 18 to 31 , two left-handed) were fit for analysis. All had a normal or corrected-to-normal vision and were naive to the purpose of the study. This experiment was approved by the ethics board of the School of Human Sciences of Osaka University. All participants gave written informed consent before participating.

\section{Materials and Procedure}

All participants performed a computer-based same/ different mental rotation task consisting of two blocks for nonsense and human-like conditions and then completed two timed paper-and-pencil tests for assessing spatial perspective-taking ability-the Road Map Test (Money et al., 1965, modified by Zacks et al., 2000) and the Spatial Orientation Test (Kozhevnikov \& Hegarty, 2001, modified by Hegarty \& Waller, 2004), in this order.

\section{Mental Rotation Task}

Stimuli were images of a three-dimensional object consisting of ten cubes. For the human-like condition, a human head was drawn on the object's uppermost cube so that it resembled a human body (Figure 1, right). For the control condition, we created a nonsense object by replacing the head of the human-like object with a nonsense pattern (Figure 1, left). For same-different comparisons, we also created mirror-reversed versions of the nonsense and human-like objects. By rotating these four images by $0^{\circ}, \pm 60^{\circ}, \pm 120^{\circ}$, and $180^{\circ}$ in the picture plane, we generated 24 stimulus images (https://doi.org/10.17605/osf.io/

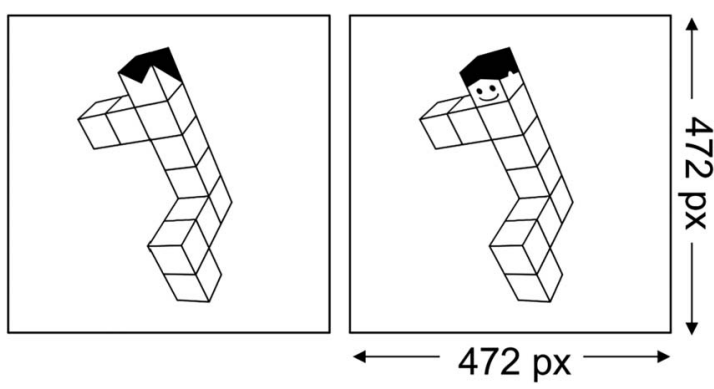

Figure 1. Stimulus images of nonsense (left) and human-like (right) objects used in the mental rotation task. The frames surrounding the images were not shown in the actual experiment.

gxnwv). All stimuli were displayed on a white background on a 21.5 " LCD monitor with a resolution of $1,920 \times 1,080$ pixels. The participants were free to choose their most comfortable viewing distance (the possible range was approximately $50-70 \mathrm{~cm}$ ).

Participants underwent the nonsense and human-like conditions in separate blocks in a counterbalanced order. In each trial, a pair of stimulus images were presented side-by-side. One of the two images was a reference object, which was always presented at $0^{\circ}$ (i.e., orientation in Figure 1), and the other was identical to or the mirrorreversed version of the object, presented either at $0^{\circ}, \pm 60^{\circ}$, $\pm 120^{\circ}$, or $180^{\circ}$. Participants had to press the "J" or "F" key as accurately and quickly as possible, according to whether the two images showed identical or different objects, respectively. The inter-trial interval was $500 \mathrm{~ms}$. Each block consisted of 192 trials: six angles $\times$ two versions of the reference object (original or mirror-reversed) $\times$ two presentation positions of the reference object (left or right) $\times$ two pair types (same or different) $\times$ four repetitions. The trial order was randomized, with no feedback given. Participants were given the opportunity to take a break after every 24 trials. Before the experimental trials, the participants completed 96 practice trials that used objects with no patterns.

\section{Psychometric Test for Assessing Spatial Perspective-Taking Ability}

The Road Map Test (Money et al., 1965, modified by Zacks et al., 2000) shows a bird's-eye diagram of a city on a page. The participants were asked to imagine walking along a route on the map and indicate whether they should turn right or left at each of the 36 corners by writing either “み” or “ひ," respectively (instead of “R” and "L," we chose the initial letters of the Japanese words for "right" and "left" because all participants were Japanese). They were not allowed to rotate the page. The Road Map Test score was the number of corners that were answered correctly within $30 \mathrm{~s}$. 
Meanwhile, the Spatial Orientation Test (Kozhevnikov \& Hegarty, 2001, modified by Hegarty \& Waller, 2004) consists of 12 items (each printed on a page). Each item presented the participants with a picture of an array of objects. The participants were instructed to imagine standing in the location of one object, facing another object, and indicate the direction to a third object by drawing an arrow in a circle printed at the bottom following a sentence below the array (e.g., "Imagine you are standing at the car and facing the traffic light. Point to the stop sign."). For the present study, we translated the original sentences into Japanese. We prohibited the participants from rotating the page or making any marks on the array. They were given 5 min to complete the test. We then calculated the absolute angular deviation from the correct answer (ranging from $0^{\circ}$ to $180^{\circ}$ ) per item and then averaged the angular deviations across items. An angular deviation of $90^{\circ}$ (chance level) was assigned to an unanswered item (only for one item of one participant).

\section{Data Processing}

We calculated the mean RTs of the mental rotation task for each combination of participant, condition (nonsense or human-like), and angular disparity $\left(0^{\circ}, 60^{\circ}, 120^{\circ}\right.$, or $\left.180^{\circ}\right)$ using data from same-pair trials. Before calculating the means, we excluded data from incorrect responses $(7.28 \%$ of the same-pair trials) and responses slower than the mean RT plus three SDs per participant $(2.00 \%$ of samepair trials). As an index of spatial perspective-taking ability, we calculated a composite score by averaging the $z$-transformed Road Map Test score and the angular deviation of the Spatial Orientation Test that was also $z$-transformed and then negated so that higher values represent greater spatial perspective-taking ability. Raw data and scripts for analysis are available at https://doi. org/10.17605/osf.io/gxnwv.

\section{Results}

\section{Preliminary Analyses}

The mean RTs of the mental rotation task are shown in Figure 2. A repeated measures ANOVA, ${ }^{1}$ whose factors were condition (nonsense or human-like) and angular disparity $\left(0^{\circ}, 60^{\circ}, 120^{\circ}\right.$, or $\left.180^{\circ}\right)$, revealed faster mean RTs for the human-like object $(M=1,088 \mathrm{~ms})$ than for the nonsense object $(M=1,280 \mathrm{~ms}), F(1,42)=28.48, \eta_{\mathrm{p}}{ }^{2}=.404$,

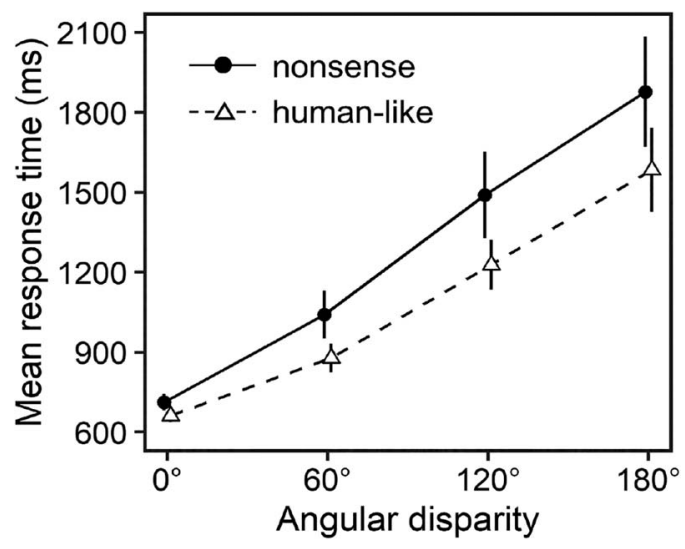

Figure 2. Mean response times of the mental rotation task $(N=43)$. Error bars represent $95 \% \mathrm{Cls}$ of means.

$p<.001$, which ensured the presence of the human-body advantage. The analysis also revealed a significant main effect of angular disparity, $F(1.44,60.43)=139.63, \eta_{\mathrm{p}}{ }^{2}=$ $.769, p<.001$, and a significant interaction between condition and angular disparity, $F(2.30,96.40)=13.10, \eta_{\mathrm{p}}{ }^{2}=$ $.238, p<.001$. These results reflected linear RT-angle functions and a shallower RT slope for the human-like object $(5.20 \mathrm{~ms} /$ degree$)$ than for the nonsense objects (6.57 ms/degree), consistent with previous studies (e.g., Amorim et al., 2006). For the following correlation analyses, we used the mean RTs of the nonsense and humanlike conditions that collapsed across angular disparities. These RTs were positively correlated with each other, $r=.767, t(41)=7.66, p<.001$ (two-tailed).

Road Map Test scores $(M=13.3, S D=4.9)$ were negatively correlated with angular deviations of the Spatial Orientation Test $\left(M=23.4^{\circ}, S D=8.4\right), r=-.388$, $t(41)=-2.70, p=.010$ (two-tailed). This implies that the two measures commonly used spatial perspective-taking ability and justified using a composite score as a more reliable and valid measure of such an ability.

\section{Relationship Between Mental Rotation and Spatial Perspective-Taking Ability}

Figure 3 shows the relationship between spatial perspective-taking score (a composite score of the Road Map Test and the Spatial Orientation Test) and mean RT for mental rotation. Correlation analyses showed that higher spatial perspective-taking scores were significantly associated with shorter RTs for both the

We have reported degrees of freedom and $p$ values corrected by Chi-Muller's $\varepsilon$ to guard against sphericity violations. 


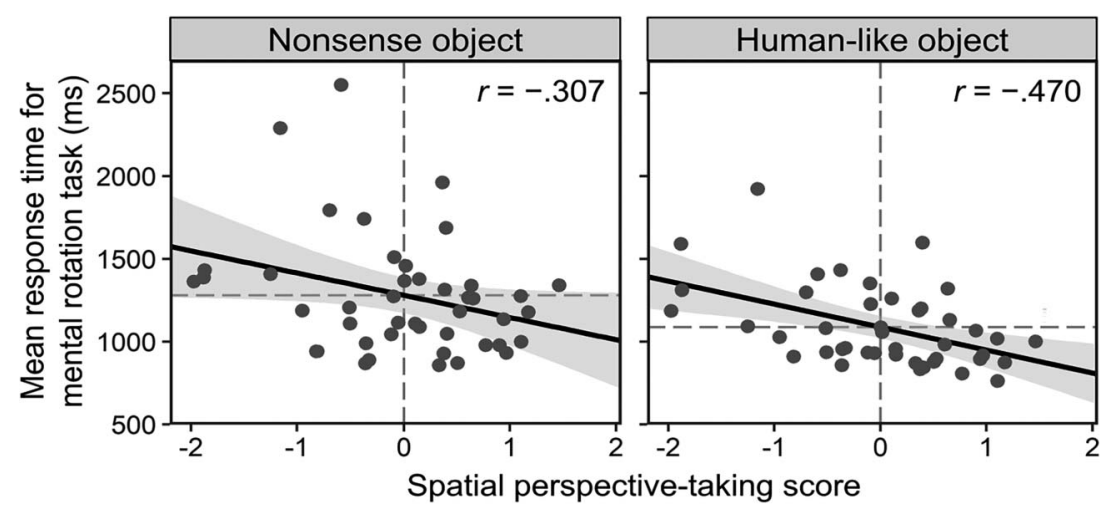

Figure 3. Relations between the spatial perspective-taking score to the mean response times for the mental rotation of the nonsense (left) or human-like (right) object $(N=43)$. Solid lines and error bands represent regression lines and their 95\% Cls. Dashed lines represent means of measures. nonsense, $r=-.307, t(41)=-2.07, p=.045$ (two-tailed), and the human-like condition, $r=-.470, t(41)=-3.41$, $p=.001$ (two-tailed), of the mental rotation task. To test our directional hypothesis that RTs for the human-like condition are more strongly associated with spatial perspective-taking ability than RTs for the nonsense condition, we compared the two correlation coefficients using Meng et al.'s (1992) method. Consistent with our hypothesis, the analysis showed that spatial perspectivetaking scores had a stronger association with RTs for human-like than for nonsense conditions, $t(40)=1.72$, $p=.046$ (one-tailed).

\section{Discussion}

To explore whether the process of mapping one's body axes onto stimuli contributes to the human-body advantage in mental rotation, we investigated the relationship between spatial perspective-taking ability and RTs for the same/different judgment of nonsense and human-like objects. First, the present study replicated the humanbody advantage reported in the literature (Amorim et al., 2006; Jansen et al., 2012; Krüger et al., 2014; Makinae \& Kasai, 2017; Makinae, et al., 2015; Muto \& Nagai, 2020; Sayeki, 1981; Voyer \& Jansen, 2016). As a novel contribution, we found that the human-body advantage occurred even when differences between the human-body and control conditions were much smaller than the previous studies. Second, and more importantly, correlation analyses revealed that spatial perspective-taking ability had a stronger association with mental rotation performance for human-like objects than for nonsense ones. These findings conform to the notion that human-like objects elicit an efficient egocentric rotation strategy in the same/different mental rotation task, leading to the human-body advantage. Although our correlational results were only a necessary condition for spatial embodiment and should be cautiously interpreted, they provided the rationale for more detailed investigations.

\section{Mediation by Egocentric Mental Rotation}

The present study provided the first evidence for the adoption of egocentric mental rotation in the same/ different judgment of human-like objects. The idea that egocentric mental rotation is elicited, even for a same/different mental rotation task, seems inconsistent with previous evidence suggesting that object-based and egocentric mental rotation strategies are adopted selectively for the same/different judgment of a pair of rotated human bodies and the left/right judgment of a single rotated human body, respectively (e.g., Habacha et al., 2018; Voyer et al., 2017; Zacks et al., 2000; Zacks \& Tversky, 2005). For example, RTs for left/right judgment were shorter and less dependent on stimulus orientation (e.g., Habacha et al., 2018; Voyer et al., 2017; Zacks et al., 2000; Zacks \& Tversky, 2005), more associated with spatial perspective-taking ability, and less associated with object-based mental rotation ability, as measured by Vandenberg and Kuse's (1978) Mental Rotation Test (Zacks et al., 2000), and differently affected by stimulus size (Habacha et al., 2018) compared to the same/ different judgment, all of which supported the task dependence of the mental rotation strategy. More critically, Zacks and Tversky (2005) demonstrated how performance on the same/different judgment of human bodies deteriorated when participants were instructed to use egocentric mental rotation as opposed to when they were instructed to use object-based mental rotation or when no instruction was given. Nonetheless, people may flexibly switch strategies (e.g., they could execute egocentric mental rotation only in trials in which it is effective) or combine both object-based and egocentric mental rotation within a trial rather than rely on a single strategy. Also, only people who are good at egocentric mental 
rotation may benefit from spatial embodiment, while others may persist in object-based mental rotation. Although these accounts are still speculative and premature, the present study suggests that human likeness of stimuli may elicit egocentric mental rotation even in the same/different task.

An alternative account is that general spatial ability, rather than spatial perspective-taking ability, contributes to the human-body advantage, given correlations among different spatial abilities (e.g., Hegarty \& Waller, 2004; Kozhevnikov \& Hegarty, 2001). The present findings alone cannot rule out this possibility because we measured only spatial perspective-taking ability using the Road Map Test and the Spatial Orientation Test. However, this alternative seems less plausible because the object-based mental rotation score (measured by Vandenberg and Kuse's Mental Rotation Test) and the spatial perspective-taking score (measured by the Road Map Test and the Spatial Orientation Test) distinctively accounted for performance on the same/different and left/right judgment tasks (Zacks et al., 2000) and because lower object-based mental rotation performance was associated with a larger human-body advantage (Makinae \& Kasai, 2017). Thus, the general spatial ability proposition may not adequately explain the present results.

\section{Limitations}

The present study has three limitations. First, since the present experiment used only cube stimuli to which a nonsense or human-like pattern was added, it is still unclear to which extent the present findings can be generalized to other stimuli, including more realistic human bodies and familiar objects other than human bodies. Recently, Muto and Nagai (2020) found that not only human-like objects but also snake-shaped cubes with a snake face can be rotated quickly (seemingly via spatial embodiment), implying that the facilitation effect is not limited to human bodies. Given that the bodily projections are feasible for any object with at least two asymmetrical intrinsic axes (Muto et al., 2019), the spatial perspectivetaking ability could contribute to a mental rotation task with other kinds of stimuli.

Second, since the present study targeted only female participants, potential sex differences in spatial embodiment were unexplored. In particular, given sex differences in mental rotation strategies (e.g., Heil \& Jansen-Osmann, 2008), and their potential effects on the human-body advantage (e.g., Makinae et al., 2015; Makinae \& Kasai, 2017; Voyer \& Jansen, 2016), we cannot rule out that the mechanisms of the human-body advantage differ across sexes, necessitating further investigations including both sexes. However, recent evidence indicates that observed sex differences in mental rotation tasks can be explained largely by psychosocial and methodological factors such as gender stereotypes, motivation, task and item structures, and participants' academic specialization (e.g., Boone \& Hegarty, 2017; Moè, 2016; Sanchis-Segura et al., 2018). Therefore, the present findings seem likely to hold for both sexes as long as such factors are properly controlled.

Third, the extent to which spatial embodiment contributes to an overall human-body advantage remains unexplained. Given the involvement of whole-body motor simulation in bodily projections entailed by spatial perspective-taking (e.g., Kessler \& Thomson, 2010; Muto et al., 2018), a spatial embodiment might not be independent of (or interact with) motoric embodiment or mental emulation of the posture of stimuli. To address this issue, future research should conduct more sophisticated experiments isolating the spatial and motoric embodiment components.

\section{Implications for Neurocognitive Research}

The present findings are expected to play an important role in deepening our understanding of the neural substrates involved in visuospatial transformations and body representations. In a recent event-related potential study, Jansen et al. (2020) found time-dependent distinctions of brain activations in frontal, central, and parietal regions between real human bodies and abstract cube objects, using the same stimuli as used by Amorim et al. (2006). Although their findings are interesting, it is still unclear which feature of stimuli is critical for the human-body advantage and distinctive brain activations (e.g., contour smoothness, luminance contrast, presence of spatial cues, and/or human-likeness). This limitation may be overcome by the present minimal stimulus manipulation (cf. Perruchoud et al., 2016, who showed that small visual differences can trigger different brain activations in another mental rotation task). More specifically, considering the role of egocentric mental rotation in the human-body advantage, neural activities for same/different judgments of human-like and nonsense objects are predicted to overlap with the contrasts between object-based and egocentric mental rotation, respectively. While previous studies on neural distinctions between object-based and egocentric mental rotation yielded somewhat mixed results, probably due to methodological differences, candidate regions responsible for egocentric mental rotation are the left parietal-temporal-occipital junction and the right parieto-occipital sulcus extending into the retrosplenial cortex (e.g., Lambrey et al., 2012; Wraga et al., 2005; for a review, see also Zacks \& Michelon, 2005). Larger 
activation in such regions can be expected to be associated with shorter overall RT and/or a shallower slope for same/ different judgments of human-like objects.

From an applied perspective, the present findings are potentially useful for clinical assessments and interventions, because mental rotation is a helpful tool to assess neuropsychological deficits and evaluate interventions (e.g., Lee et al., 1998; Scandola et al., 2019). Specifically, the simple inclusion of objects with human-like features would allow us to ameliorate visuospatial assessments by considering a patient's spatial perspective-taking ability and to establish customized therapeutic approaches according to each patient's mental spatial ability. Relatedly, our recent work (Muto et al., 2020) administered a paperand-pencil mental rotation test with almost the same objects as the present study to elderly people aged 86-97 years and revealed that performance for human-like objects was better and more preserved with age than for nonsense objects. This suggests a neural basis for the mental rotation of human-like objects that is partially different from that of the mental rotation of nonsense objects in a way that compensates for the age-related decline of the object-based mental rotation ability. On the basis of the present findings, further studies are needed to establish the neuropsychological significance of the human-body advantage.

\section{References}

Amorim, M.-A., Isableu, B., \& Jarraya, M. (2006). Embodied spatial transformations: "body analogy" for the mental rotation of objects. Journal of Experimental Psychology: General, 135(3), 327-347. https://doi.org/10.1037/0096-3445.135.3.327

Boone, A. P., \& Hegarty, M. (2017). Sex differences in mental rotation tasks: Not just in the mental rotation process! Journal of Experimental Psychology: Learning, Memory, and Cognition, 43(7), 1005-1019. https://doi.org/10.1037/xlm0000370

Habacha, H., Moreau, D., Jarraya, M., Lejeune-Poutrain, L., \& Molinaro, C. (2018). Dissociating object-based from egocentric transformations in mental body rotation: Effect of stimuli size. Experimental Brain Research, 236(1), 275-284. https://doi.org/ 10.1007/s00221-017-5125-y

Hegarty, M., \& Waller, D. (2004). A dissociation between mental rotation and perspective-taking spatial abilities. Intelligence, 32(2), 175-191. https://doi.org/10.1016/j.intell.2003.12.001

Heil, M., \& Jansen-Osmann, P. (2008). Sex differences in mental rotation with polygons of different complexity: Do men utilize holistic processes whereas women prefer piecemeal ones? The Quarterly Journal of Experimental Psychology, 61(5), 683-689. https://doi.org/10.1080/17470210701822967

Huttenlocher, J., \& Presson, C. C. (1973). Mental rotation and the perspective problem. Cognitive Psychology, 4(2), 277-299. https://doi.org/10.1016/0010-0285(73)90015-7

Jansen, P., Lehmann, J., \& Van Doren, J. (2012). Mental rotation performance in male soccer players. PLoS One, 7(10), e48620. https://doi.org/10.1371/journal.pone.0048620
Jansen, P., Render, A., Scheer, C., \& Siebertz, M. (2020). Mental rotation with abstract and embodied objects as stimuli: Evidence from event-related potential (ERP). Experimental Brain Research, 238(3), 525-535. https://doi.org/10.1007/s00221-02005734-w

Kessler, K., \& Thomson, L. A. (2010). The embodied nature of spatial perspective taking: Embodied transformation versus sensorimotor interference. Cognition, 114(1), 72-88. https://doi.org/10. 1016/j.cognition.2009.08.015

Kozhevnikov, M., \& Hegarty, M. (2001). A dissociation between object manipulation spatial ability and spatial orientation ability. Memory \& Cognition, 29(5), 745-756. https://doi.org/10.3758/ BF03200477

Krüger, M., Amorim, M.-A., \& Ebersbach, M. (2014). Mental rotation and the motor system: Embodiment head over heels. Acta Psychologica, 145(1), 104-110. https://doi.org/10.1016/j.actpsy. 2013.11.004

Lambrey, S., Doeller, C., Berthoz, A., \& Burgess, N. (2012). Imagining being somewhere else: Neural basis of changing perspective in space. Cerebral Cortex, 22(1), 166-174. https://doi.org/10.1093/ cercor/bhr101

Lee, A. C., Harris, J. P., \& Calvert, J. E. (1998). Impairments of mental rotation in Parkinson's disease. Neuropsychologia, 36(1), 109-114. https://doi.org/10.1016/S0028-3932(97)00017-1

Makinae, S., \& Kasai, T. (2017). Mentarurōtēshon ni okeru shintai e no ruisui to seisa [Body analogy and sex differences in mental rotation]. Shinri-gaku kenkyū, 88(5), 452-459. https://doi.org/10. 4992/jjpsy.88.16053

Makinae, S., Yamazaki, K., \& Kasai, T. (2015). Mentarurōtēshon kadai ni okeru shintai-ka sa reta ninchi to seisa. [Embodied cognition and sex differences in a mental rotation task]. Denshi jōhō gakkai gijutsu kenkyū hōkoku, 114, 81-84.

Meng, X.-l., Rosenthal, R., \& Rubin, D. B. (1992). Comparing correlated correlation coefficients. Psychological Bulletin, 111(1), 172-175. https://doi.org/10.1037/0033-2909.111.1.172

Moè, A. (2016). Teaching motivation and strategies to improve mental rotation abilities. Intelligence, 59, 16-23. https://doi.org/ 10.1016/j.intell.2016.10.004

Money, J., Alexander, D., \& Walker, H. T. (1965). A standardized road-map test of direction sense: manual. Johns Hopkins Press.

Muto, H., Gondo, Y., Inagaki, H., Masui, Y., Nakagawa, T., Ogawa, M., Onoguchi, W., Ishioka, Y., Numata, K., \& Yasumoto, S. (2020). Human-body analogy improves mental rotation performance in people aged 86 to 97 years. https://doi.org/10.31234/osf.io/ n3fbc

Muto, H., Matsushita, S., \& Morikawa, K. (2018). Spatial perspective taking mediated by whole-body motor simulation. Journal of Experimental Psychology: Human Perception and Performance, 44(3), 337-355. https://doi.org/10.1037/xhp0000464

Muto, H., Matsushita, S., \& Morikawa, K. (2019). Object's symmetry alters spatial perspective-taking processes. Cognition, 191, 103987. https://doi.org/10.1016/j.cognition.2019.05.024

Muto, H., \& Nagai, M. (2020). Mental rotation of cubes with a snake face: The role of the human-body analogy revisited. Visual Cognition, 28(2), 106-111. https://doi.org/10.1080/13506285. 2020.1727598

Perruchoud, D., Michels, L., Piccirelli, M., Gassert, R., \& Ionta, S. (2016). Differential neural encoding of sensorimotor and visual body representations. Scientific Reports, 6(1), 37259. https://doi. org/10.1038/srep37259

Sanchis-Segura, C., Aguirre, N., Cruz-Gómez, Á. J., Solozano, N., \& Forn, C. (2018). Do gender-related stereotypes affect spatial performance? Exploring when, how and to whom using a chronometric two-choice mental rotation task. Frontiers in Psychology, 9, 1261. https://doi.org/10.3389/ fpsyg.2018.01261 
Sayeki, Y. (1981). "Body analogy" and the cognition of rotated figures. Quarterly Newsletter of the Laboratory of Comparative Human Cognition, 3(2), 36-40.

Scandola, M., Dodoni, L., Lazzeri, G., Arcangeli, C. A., Avesani, R., Moro, V., \& Ionta, S. (2019). Neurocognitive benefits of physiotherapy for spinal cord injury. Journal of Neurotrauma, 36(12), 2028-2035. https://doi.org/10.1089/neu.2018.6123

Searle, J. A., \& Hamm, J. P. (2017). Mental rotation: An examination of assumptions. Wiley Interdisciplinary Reviews: Cognitive Science, 8(6), e1443. https://doi.org/10.1002/wcs.1443

Shepard, R. N., \& Metzler, J. (1971). Mental rotation of threedimensional objects. Science, 171(3972), 701-703. https://doi. org/10.1126/science.171.3972.701

Tarampi, M. R., Heydari, N., \& Hegarty, M. (2016). A tale of two types of perspective taking. Psychological Science, 27(11), 1507-1516. https://doi.org/10.1177/0956797616667459

Vandenberg, S. G., \& Kuse, A. R. (1978). Mental rotations, a group test of three-dimensional spatial visualization. Perceptual and Motor Skills, 47(2), 599-604. https://doi.org/10.2466/pms.1978. 47.2.599

Voyer, D., \& Jansen, P. (2016). Sex differences in chronometric mental rotation with human bodies. Psychological Research, 80(6), 974-984. https://doi.org/10.1007/s00426-015-0701-x

Voyer, D., Jansen, P., \& Kaltner, S. (2017). Mental rotation with egocentric and object-based transformations. The Quarterly Journal of Experimental Psychology, 70(11), 2319-2330. https:// doi.org/10.1080/17470218.2016.1233571

Voyer, D., Voyer, S., \& Bryden, M. P. (1995). Magnitude of sex differences in spatial abilities: A meta-analysis and consideration of critical variables. Psychological Bulletin, 117(2), 250-270. https://doi.org/10.1037/0033-2909.117.2.250

Wraga, M., Creem, S. H., \& Proffitt, D. R. (2000). Updating displays after imagined object and viewer rotations. Journal of Experimental Psychology: Learning, Memory, and Cognition, 26(1), 151-168. https://doi.org/10.1037//0278-7393.26.1.151

Wraga, M., Shephard, J. M., Church, J. A., Inati, S., \& Kosslyn, S. M. (2005). Imagined rotations of self versus objects: An fMRI study. Neuropsychologia, 43(9), 1351-1361. https://doi.org/10.1016/j. neuropsychologia.2004.11.028

Zacks, J. M., \& Michelon, P. (2005). Transformations of visuospatial images. Behavioral and Cognitive Neuroscience Reviews, 4(2), 96-118. https://doi.org/10.1177/1534582305281085
Zacks, J. M., Mires, J., Tversky, B., \& Hazeltine, E. (2000). Mental spatial transformations of objects and perspective. Spatial Cognition and Computation, 2(4), 315-332. https://doi.org/10. 1023/A:1015584100204

Zacks, J. M., \& Tversky, B. (2005). Multiple systems for spatial imagery: Transformations of objects and bodies. Spatial Cognition \& Computation, 5(4), 271-306. https://doi.org/10.1207/ s15427633scc0504_1

\section{History}

Received October 24, 2020

Revision received January 26, 2021

Accepted February 7, 2021

Published online April 12, 2021

\section{Conflict of Interest}

The author declares no conflicts of interest with respect to the authorship or the publication of this article.

\section{Publication Ethics}

This experiment was approved by the ethics board of the School of Human Sciences of Osaka University.

\section{Open Data}

Raw data, $\mathrm{R}$ scripts, and materials used in this paper are available at https://doi.org/10.17605/osf.io/gxnwv.

\section{Funding}

This work was supported by a JSPS Grant-in Aid for JSPS Fellows (Number 19J00072)

\section{ORCID}

Hiroyuki Muto

(iD) https://orcid.org/0000-0002-0007-6019

\section{Hiroyuki Muto}

Kokoro Research Center

Kyoto University

46 Shimoadachi-cho

Yoshida, Sakyo-ku

Kyoto 606-8501

Japan

h.muto@zm.commufa.jp 